\section{Synthetic nacre by predesigned matrix-directed mineralization}

\author{
Li Bo Mao, ${ }^{1,2}$ Huai Ling Gao, ${ }^{1,2}$ Hong Bin Yao, ${ }^{1,2}$ Lei Liu, ${ }^{1,2,3}$ Helmut Cölfen, ${ }^{3}$ \\ Gang Liu, ${ }^{4}$ Si Ming Chen, ${ }^{1,2}$ Shi Kuo Li, ${ }^{1,2}$ You Xian Yan, ${ }^{1,2}$ \\ Yang Yi Liu, ${ }^{1,2}$ Shu Hong Yu ${ }^{1,2,4 *}$
}

Although biomimetic designs are expected to play a key role in exploring future structural materials, facile fabrication of bulk biomimetic materials under ambient conditions remains a major challenge. Here, we describe a mesoscale "assembly-and-mineralization" approach inspired by the natural process in mollusks to fabricate bulk synthetic nacre that highly resembles both the chemical composition and the hierarchical structure of natural nacre. The millimeter-thick synthetic nacre consists of alternating organic layers and aragonite platelet layers (91 weight percent) and exhibits good ultimate strength and fracture toughness. This predesigned matrix-directed mineralization method represents a rational strategy for the preparation of robust composite materials with hierarchically ordered structures, where various constituents are adaptable, including brittle and heat-labile materials.

B iological materials are built from limited components, but their mechanical perfor mances, such as strength and toughness, are far beyond their artificial counterparts. The secret of success is their hierarchically ordered structure at multiscale levels $\left(\begin{array}{ll}1 & 4\end{array}\right)$. The most studied model among these biological ma terials is the nacreous part in some mollusk shells that consists of about 95 weight $\%$ (wt \%) of brittle aragonitic $\mathrm{CaCO}_{3}$ and $5 \mathrm{wt} \%$ of organic materials (5). Mollusks produce nacre by first generating several layers of insoluble $\beta$ chitin matrix filled with silk fibroin gel (6). Then aragonite cores form on the surface of the matrix at the nuclea tion sites (7), followed by lateral growth in the confined space of adjacent organic layers, which finally leads to a Voronoi pattern (5). These arago nite platelets, despite their single crystal diffraction pattern, are not perfect single crystals but essen tially consist of nanograins with the same crystal lographic orientation (mesocrystals), whereby the platelets are not as fragile as perfect single crys tals (8). The mature nacre has a "brick and mortar" microstructure where aragonite platelet layers are bound by an organic matrix (3). Through a number of such structural designs and toughening mech anisms at multiscale levels $(2,9,10)$, nacre recon ciles its toughness and strength, which are mutually exclusive in most artificial materials (II).

In contrast to biological materials, the evolu tion of synthetic structural materials has been achieved predominately by developing new syn

1Division of Nanomaterials and Chemistry, Hefei National Laboratory for Physical Sciences at the Microscale, University of Science and Technology of China, Hefei, 230026, China ${ }^{2}$ Chinese Academy of Sciences, Center for Excellence in Nanoscience, Collabordive Innovation Center of Suzhou Nano Science and Technology. Hefei Science Center, Department of Chemistry, University of Science and Technology of China, Hefei, 230026, China ${ }^{3}$ University of Konstanz, Physical Chemistry. Universitätsstraße 10, D 78457 Konstanz, Germany. ${ }^{4}$ Nationa Synchrotron Radiation Laboratory, University of Science and Technology of China, Hefei, 230029, China

*Corresponding author. Email: shyu@ustc.educn thetic compounds rather than optimizing the micro/nanostructures of existing materials. There fore, bioinspired designs of multiscale structures are promising for developing surpassing structural materials $(5,12)$. However, the fabrication of bulk biomimetic materials is by no means a low hanging fruit (12), because it is hard to balance the well organized hierarchical structure and the efficiency. For nacre, the strategies used for producing its artificial counterparts can be categorized into three groups: the layer by layer technique (13 16), the self assembly technique (17 20), and the slurry based freeze casting/magnetic field assisted slip casting and sintering technique (2I 25). Although these artificial materials are to some extent sim ilar to natural nacre, the state of the art techniques focus on mimicking the layered structure by ani sotropic assembly of building blocks but have not achieved the fabrication of bulk synthetic nacre via a mineralization strategy that is adopted by many living creatures to produce biomaterials, including nacre $(57)$. Furthermore, the high temperature heat treatment (22 25) excludes many heat labile materials and thus substantially lim its their applications.

Considering that mollusks build their nacre by the mineralization in a preformed laminated ma trix (5), we developed a consecutive assembly and mineralization process (Fig. 1) to produce synthetic nacre by a mesoscale approach where the nano structure and the microstructure are controlled simultaneously. Through a freezing induced assem bly process (Fig. 1, A and B, and fig. S1), a chitosan matrix with predesigned laminated structure is fabricated (fig. S2, A, B, D, and E). Then the matrix is acetylated (Fig. 1C) and transformed to $\beta$ chitin to avoid unwanted swelling or dissolution (fig. S2, $\mathrm{C}$ and $\mathrm{E}$, and table $\mathrm{S1}$ ). The acetylated matrix is mineralized in a peristaltic pump driven circula tory system via the decomposition of $\mathrm{Ca}\left(\mathrm{HOO}_{3}\right)_{2}$ in the presence of polyacrylic acid (PAA) and $\mathrm{Mg}^{2+}$ (Fig. 1D). Then the final material is obtained by silk fibroin infiltration and hot pressing of the miner alized matrix. The thickness of the bulk synthetic nacre is about 1 to $2 \mathrm{~mm}$, based on the thickness of the original chitin matrix (Fig. 2B), which can be further increased by using thicker matrix (Fig. 1B). The $\mathrm{CaOO}_{3}$ in the synthetic nacre is aragonite (fig. S3), which is attributed to the control of the additives (26).

Typically, as the size of the three dimensional (3D) matrix increases, the mass transfer throughout
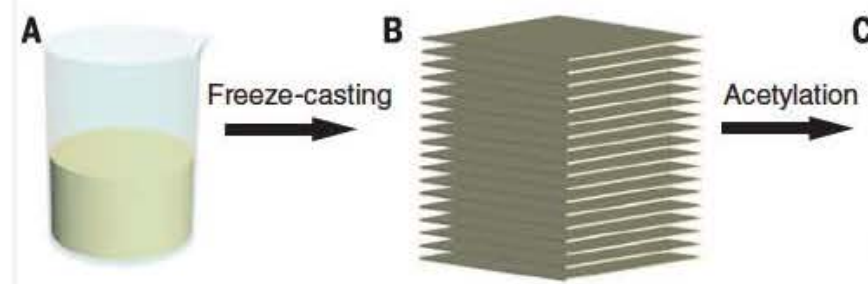

E Hot-pressing at $80^{\circ} \mathrm{C}$

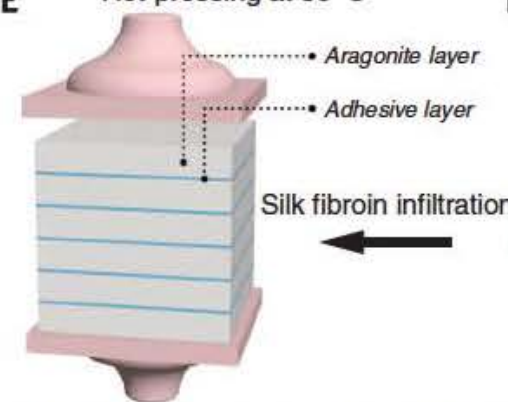

D

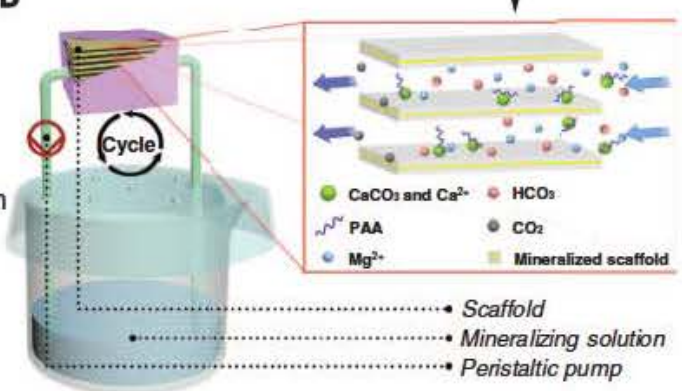

Fig. I Fabrication scheme of the synthetic nacre. (A) Starting solution, chitosan/acetic acid solution. (B) Freeze-casted laminated chitosan matrix. (C) Matrix after acetylation, where chitosan is converted to $\beta$-chitin. (D) Mineralization of the matrix. Fresh mineralizing solution is pumped to flow through the space between the layers in the matrix, bringing in $\mathrm{Ca}^{2+}, \mathrm{Mg}^{2+}, \mathrm{HCO}_{3}{ }^{-}$, and PAA for mineralization and taking out excess $\mathrm{CO}_{2} . \mathrm{CaCO}_{3}$ precipitates onto the layers and $\mathrm{CO}_{2}$ diffuses into the air through the pin holes in the paraffin film. (E) Laminated synthetic nacre is obtained after silk fibroin infiltration and hot-pressing. 
the matrix will be more difficult, which means the mineralization of the whole matrix will be re tarded. In our work, this problem is overcome through experimental designs. There is enough space between the chitin layers of the matrix, which is indispensable for the mass transfer across the whole laminated structure and thus facilitates the thorough mineralization of the matrix. Other wise, a dense mineral shell will form on the surface and obstruct the mineralization process inside (fig. S4A). To promote the mass transfer in the matrix further, the precipitation of $\mathrm{CaCO}_{3}$ is achieved by the decomposition of calcium bicarbonate rather than the gas diffusion method because the sponta neous diffusion of $\mathrm{CO}_{2}$ (fig S4B) is much slower than the direct pump driven injection of $\mathrm{Ca}\left(\mathrm{HOO}_{3}\right)_{2}$ (fig. S4, C and D). Consequently, the whole matrix mineralizes to the extent that the resulting syn thetic nacre contains as much as $91 \mathrm{wt} \%$ of $\mathrm{CaCO}_{3}$, which is quite comparable to natural nacre (5). Be cause of the high inorganic content, the synthetic nacre exhibits high stability in water without notice able swelling (movie S1). More important, while the growth of natural nacre takes months or even years, only 2 weeks are needed for the preparation of the bulk synthetic nacre whose composition is almost the same as natural nacre (figs. S3 and S5) (5).

The synthetic nacre shares striking similarities with natural nacre from the shell of mollusk such as Anodonta woodiana. The fracture surface of the bulk synthetic nacre reveals a laminated nacre like microstructure (Fig. 2, A to D; fig. S6, A and $\mathrm{B}$; and movie S2). The thickness of the alternating aragonite and organic layers is 2 to $4 \mu \mathrm{m}$ and 100 to $150 \mathrm{~nm}$, respectively. Hence, it is supposed that the Bragg diffraction induced structural coloration (5) of the synthetic nacre is not in the visible range (Fig. 2B). Each mineral layer is made up of tilelike aragonite platelets and exhibits a structure similar to Voronoi pattern, which is typically observed in natural nacre (Fig. 2, E and F) (5). However, as the mineralization conditions are complicated and the control of the process in our experiment is not as good as the biomineralization process in mollusks, the Voronoi patterns are not so perfect in the syn thetic nacre (red circles in Fig. 2F). The formation of this pattern can be ascribed to the growth mech anism that $\mathrm{CaCO}_{3}$ selectively precipitates at some nucleation sites (white arrowheads in Fig. 2F) that were identified to be rich in carboxyl groups (7), and then these initial crystals grow laterally on the chitin layers until they meet each other to form a boundary (fig. S6C and movie $\mathrm{S} 3$ ). As the matrix gradually mineralizes, the chitin layers are assimi lated by the minerals, probably because of elec trostatic attraction (figs. S7 and S8), whereas the infiltrated silk fibroin forms the organic layers between the aragonite layers (fig. S9). Both the average size and the aspect ratio of the aragonite platelets in the synthetic nacre are significantly larger than that in A. woodiana nacre (fig. S10), which greatly affect the properties of the as fabricated nacre like materials (19).

The aragonite platelets in the synthetic nacre consist of attached nanograins with diameters ranging from 10 to $100 \mathrm{~nm}$ (Figs. $2 \mathrm{H}$ and $3 \mathrm{~A}$ ), in accordance with those in natural nacre (Fig. 2G)
(8). Further analysis of the nanograins reveals the crystallographic features of the platelets (Fig. 3, B to D). The single crystal like fast Fourier trans form (FFT) patterns (Fig. 3, C and D) of the boundary areas (Fig. 3B) indicate the orientation continuity between adjacent nanograins in a single platelet, because the precipitation of $\mathrm{CaCO}_{3}$ in our experiment should follow a nonclassical crystalli zation mechanism $(27,28)$. Although the aragonite platelets are mesocrystals (27) due to the orienta tion continuity of individual nanoparticles, the aragonite platelets in a layer grow independently (fig. S6C and movie S3), and thus the crystal orien tation degree of the whole synthetic nacre is as low as $\sim 9 \%$. Therefore, a single aragonite layer shows distinctive dark and bright mosaics under cross polarized light where the brightness of these tiles depends on their orientations (Fig. 3E and fig.
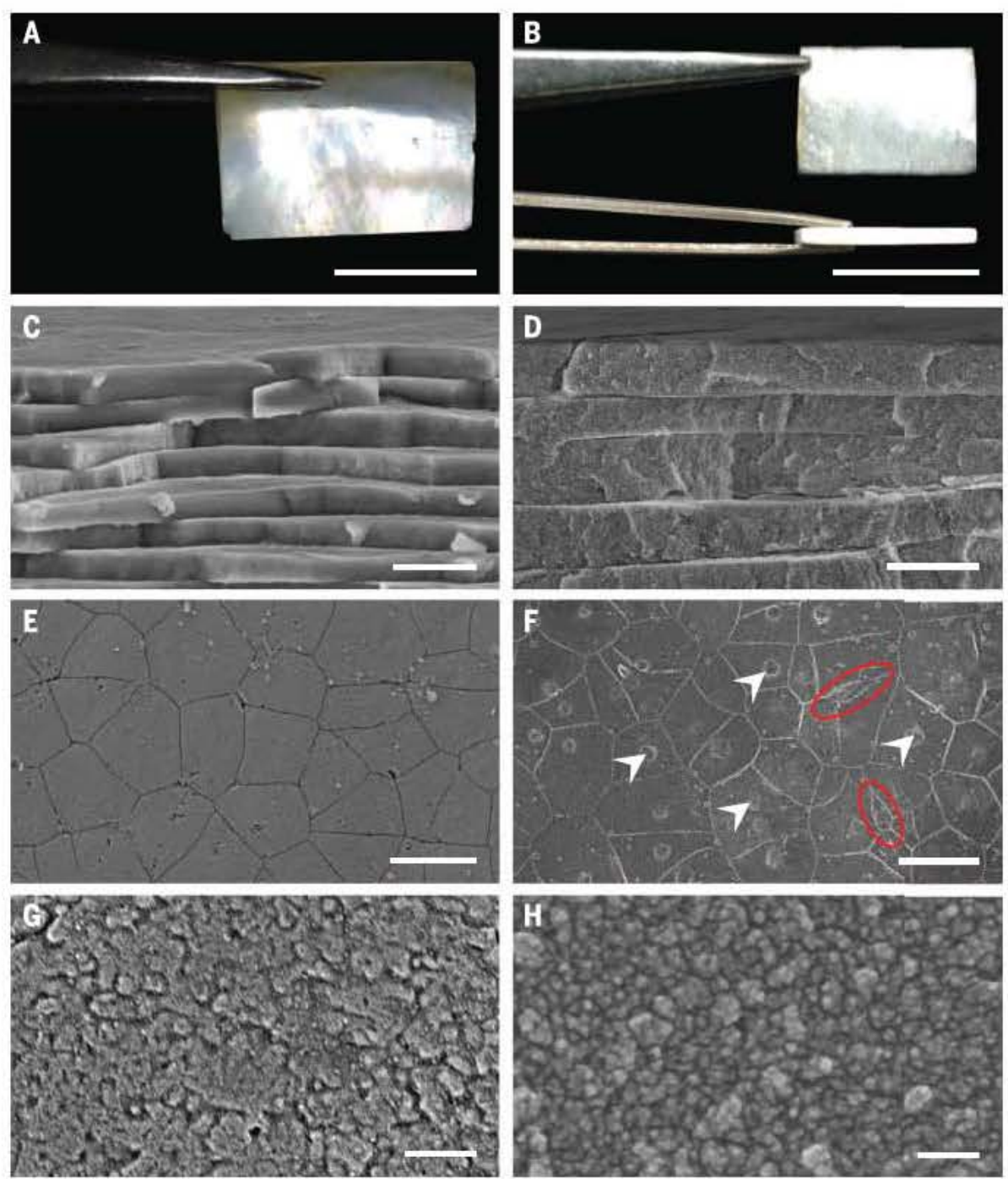

Fig. 2. The comparison of the appearance and multiscale structure between natural and synthetic nacre. (A) A. woodiana nacre. (B) Bulk synthetic nacre. (C and D) Fracture surface of (C) A. woodiana nacre and (D) synthetic nacre. (E and F) Voronoi pattern of the aragonite layer in (E) A. woodiana nacre and (F) synthetic nacre. ( $G$ and $\mathbf{H}$ ) Enlarged micrographs of the aragonite platelet of (G)A. woodiana nacre and (H) synthetic nacre. Scale bars are $1 \mathrm{~cm}, 1 \mathrm{~cm}, 3 \mu \mathrm{m}, 3 \mu \mathrm{m}, 5 \mu \mathrm{m}, 100 \mu \mathrm{m}, 100 \mathrm{~nm}$, and $100 \mathrm{~nm}$ for (A) to (H), respectively. 
be attributed to their unique connected nanograin structure (Fig. 2, G and H) (29). Like the nano grains in natural nacre that are bound by or ganics like proteins ( 8 ), it is suggested that the nanograins in the synthetic nacre (Fig. 3A) also contain or are connected by the added PAA mol ecules, which can strongly interact with $\mathrm{CaCO}_{3}$ (30). Furthermore, the assimilated chitin shreds embedded in the aragonite layers (fig. S8, C and D) provide additional binding components and act as buffer zones for internal stress (31). Con sequently, although there are weak cleavage planes in abiotic single crystals, there is no cleavage plane in these mesocrystals; the energy can be dissipated efficiently via breaking the bonding between nanograins, and the nanocracks in these mesocrystals can be localized $(8,32)$. Using the Olive Pharr model to analyze the data obtained from the NI tests, it can be found that the syn thetic nacre undergoes losses in its elastic mod ulus and hardness (fig. S11), which is ascribed mainly to the thick grain boundaries between the aragonite nanograins (Fig. 3A, fig. S12, and the calculation in the supplementary materials). It is estimated that the volume fraction of organic components in the synthetic nacre is about $14 \%$, whereas in A. woodiana nacre it is less than $8 \%$. Accordingly, the macroscopic density of the syn thetic nacre is significantly smaller than that of aragonite and $A$. woodiana nacre (fig. S13). More over, because the organic components in the grain boundaries are sensitive to water, the elastic modulus of the fully hydrated synthetic nacre decreases, whereas the ultimate strain in creases remarkably (fig. S14).

As the composition and the hierarchical struc ture of the synthetic nacre bear a striking resem blance to natural nacre, the macroscopic mechanical properties of the synthetic nacre are also supe rior to pure aragonite and its related composites and comparable to that of natural nacre (Fig. 4B; see also fig S13). The rising crack extension resist ance curves of both the synthetic and natural nacre indicate extrinsic toughening mechanisms in these materials (Fig. 4C). The reinforced performance of the synthetic nacre is attributed to the structural features at multiscale levels where the organic components play a key role (figs. S15 and S16). The assembled nanograin architecture and the organic binders (e.g, PAA and chitin) by which energy can be dissipated and nanocracks can be localized are the structural basis of the macroscopic perform ance of the synthetic nacre. Moreover, the lam inated nacre like structure leads to crack branching, crack deflection, crack blunting, crack trapping in the organic layers, and platelet bridging (Fig. 4, D and E; see also fig. S17, E and F) (33). Some microscopic features, such as platelet waviness and dovetail structure that are responsible for the hardening and damage tolerance in natural nacre (34), have also been observed in the synthetic nacre (fig. S18). In addition, the delamination process is retarded by the infiltrated silk fibroin binding layers and thus further dissipates energy (Fig. 4F; see also fig. S9, A and B). However, because their microstructures (fig. S19) as well as the suggested micromechanical models are not exactly the same
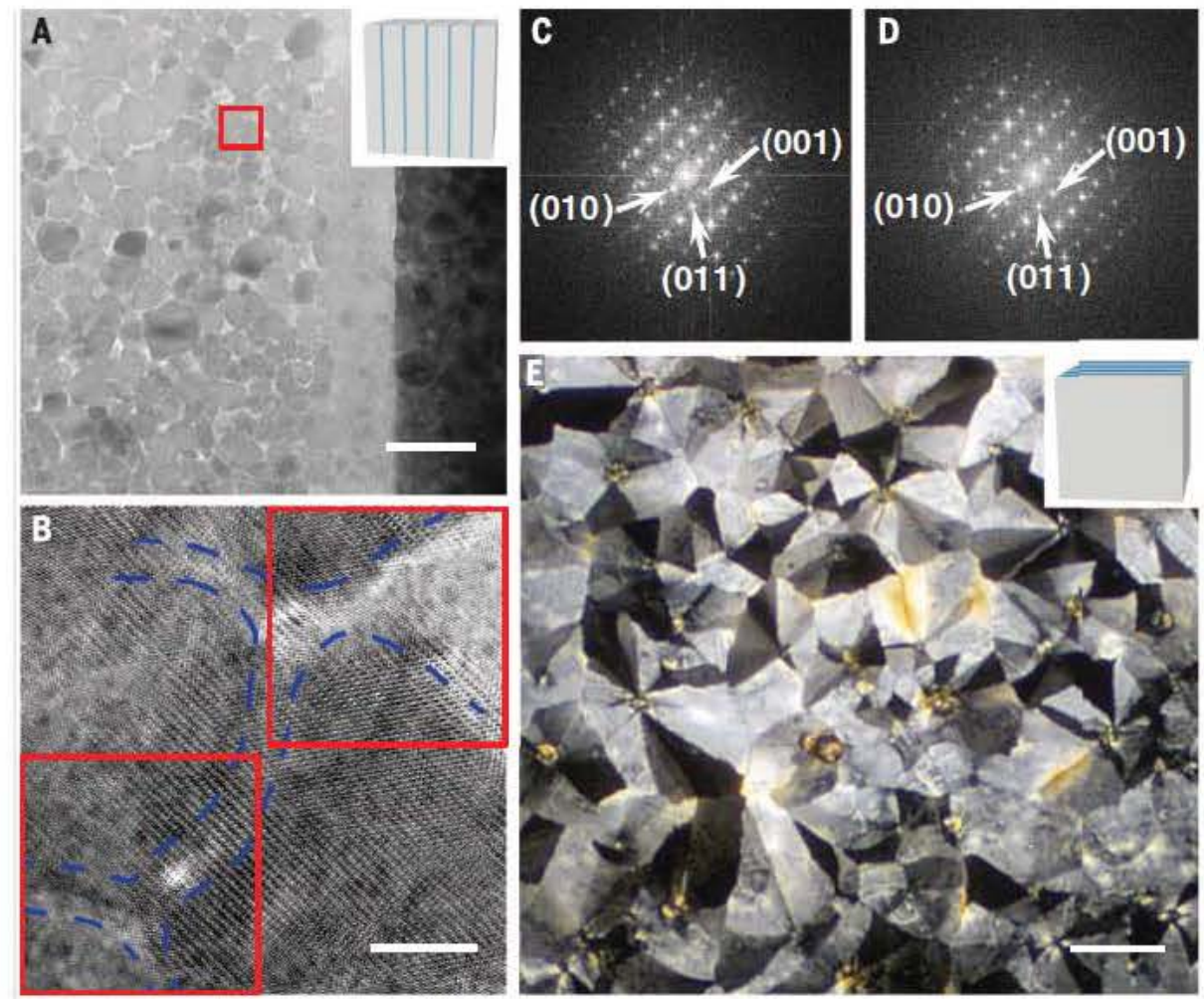

Fig. 3. Crystallographic structure of the synthetic nacre. (A) Cross-sectional transmission electron microscope (TEM) image of the synthetic nacre. The inset shows the view direction. (B) High-resolution TEM (HRTEM) image of the selected area in (A). The boundaries of thenanograins are marked with dashed blue lines. (C and D) FFT of the selected squares denoted by red lines in (B), where (C) corresponds to the top right square and (D) the bottom left. (E) Optical micrograph of the aragonite layer under crosspolarized light, where the inset shows the view direction. Scale bars are $100 \mathrm{~nm}, 10 \mathrm{~nm}$, and $50 \mu \mathrm{m}$ for (A). (B), and (E), respectively.
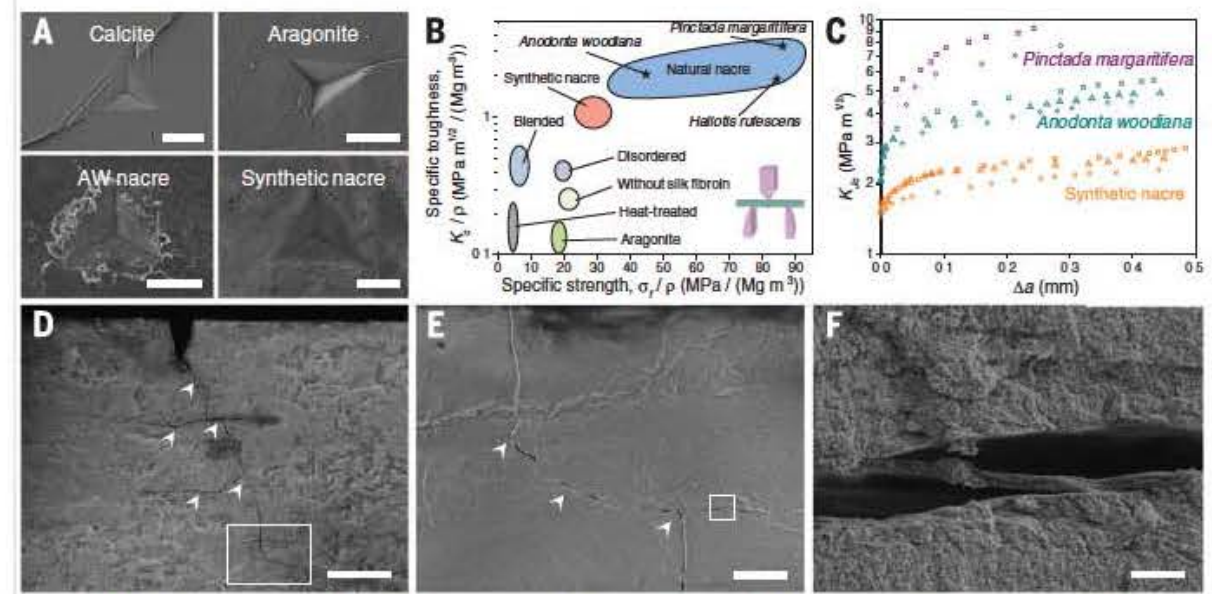

Fig. 4. Mechanical properties of the synthetic nacre. (A) Residual indents of the Berkovich diamond tip in abiotic minerals, A. woodiana (AW) nacre, and the synthetic nacre. (B) Specific fracture toughness versus specific ultimate flexural strength, illustrating the mechanical performance of the synthetic nacre, natural nacre, pure aragonite, and their related materials. (C) Rising crack-extension resistance curves (evaluated by the steady-state fracture toughness $K_{J c}$ ) of the synthetic nacre and some natural nacre. (D) Profile of the fractured synthetic nacre showing the multiple toughening mechanisms. (E) Crack deflection between layers and crack branching [enlarged micrograph of the marked area in (D)]. (F) Crackinduced interlamellar debonding in the synthetic nacre [enlargement of the marked area in (E)]. The data of Pinctada margaritifera and Haliotis rufescens are adapted from (35) and (36). Scale bars are 10, 200, 40. and $2 \mu \mathrm{m}$ for (A), (D), (E), and (F), respectively. 
(fig. S20), the mechanical properties of the syn thetic nacre are still not as good as that of natural nacre $(35,36)$ (Fig. 4, B and C). Due to the larger aspect ratio of the aragonite platelets in the syn thetic nacre, the platelets exhibit a "partly pullout" behavior, which leads to lower crack resistance capability.

Because the precipitation of the second phase onto the matrix relies on electrostatic force, $\mathrm{CaCO}_{3}$ and chitin can be substituted by other precursors with opposite charges to make superior compo sites such as engineering ceramics (21 24) (figs. S21 and S22). Besides, as the dependence of properties of the composite materials on the characteristic length of their periodic microstructure (37), the mechanical performance of these materials can be optimized by adjusting the properties of the original matrix (38), which affect both the amount of electrostatically absorbed precipitates and the density of the nucleation sites. The fabrication of the laminated synthetic nacre is not a special case; there are other techniques, such as program mable 3D printing, for constructing predesigned macroscopic matrices that can be readily incor porated with our strategy to produce composite materials. Moreover, this strategy is also adapt able for fabricating robust bulk materials with brittle and heat labile components (fig. S21B). Given the importance of nano and microscopic struc tures for the materials performance, we thus anti cipate that our method can be extended to produce various composite materials with unique properties.

\section{REFERENCES AND NOTES}

1. P. Fratzl, R. Weinkamer, Prog. Mater. Sci. 52, 12631334 (2007).

2. B. Ji, H. Gao, Annu Rev. Mater. Res. 40, 77100 (2010).

3. J. Wang, Q. Cheng, Z. Tang, Chem. Soc. Rev. 41, 11111129 (2012).

4. M. A. Meyers, J. McKittrick, P. Y. Chen, Science 339, 773779 (2013)

5. H. B. Yao, J. Ge, L. B. Mao, Y. X. Yan, S. H. Yu, Adv. Mater. 26. 163188 (2014).

6. A. G. Checa, J. H. E. Cartwright, M. G. Willinger, Proc. Natl. Acad. Sci. U.S.A. 106, 3843 (2009).

7. L. Addadi, D. Joester, F. Nudelman, S. Weiner, Chemistry 12, 980987 (2006).

8. Z. Huang, X. Li, Sci. Rep. 3, 1693 (2013).

9. P. Y. Chen et al, J. Mech. Behav. Biomed. 1, 208226 (2008).

10. L. Jager, P. Fratzl, Biophys. J. 79, 17371746 (2000).

11. R. O. Ritchie, Nat. Mater. 10, 817822 (2011).

12. U. G. K. Wegst, H. Bai, E. Saiz, A. P. Tomsia, R. O. Ritchie, Nat. Mater. 14, 2336 (2015).

13. Z. Tang, N. A. Kotov, S. Magonov, B. Ozturk, Nat. Mater. 2. 413418 (2003).

14. A. Finnemore et al, Nat. Commun. 3, 966 (2012)

15. Y. Kim et al., Nature 500, 5963 (2013).

16. T. Kato, T. Suzuki, T. Irie, Chem Lett. 29, 186187 (2000).

17. L. J. Bonderer, A. R. Studart, L J. Gauckler, Science 319 , 10691073 (2008).

18. P. Laaksonen et al., Angew Chem. int. Ed 50, 86888691 (2011).

19. P. Das et al., Nat. Commin. 6. 5967 (2015).

20. B. Zhu et al., Angew. Chem. Int. Ed. 54, 86538657 (2015).

21. H. Le Ferrand, F. Botville, T. P. Niebel, A. R. Studart, Nat. Mater. 14, 11721179 (2015).

22. S. Deville, E. Saiz, R. K Nalla, A. P. Tomsia, Science 311 515518 (2006).

23. E. Munch et al., Science 322, 15161520 (2008).

24. F. Bouvilie et al., Nat. Mater. 13, 508514 (2014).

25. H. Bai, Y. Chen, B. Delattre, A. P. Tomsia, R. O. Ritchie, Sci. Adk 1, el500849 (2015).

26. F. Zhu et al., Chem. Asian J. 8, 30023009 (2013).

27. M. Niederberger, H. Colfen, Phys. Chem Chem. Phys. 8. 32713287 (2006).

28. H. Colfen, M. Antonietti, Mesocrystals and Nonclassical Crystalization (Wiley, Chichester, UK, 2008).
9. J. Wang L L Shaw, Biomaterials 30,6565 6572 (2009).

30. D. Gebauer, H. Colfen, A. Verch, M. Antonietti, Adv. Mater. 21, 435439 (2009).

31 S. Weiner, L Addadi, H. D. Wagner, Mater. Sci. Eng. C 11, 18 (2000).

32. J. Seto et al., Proc. Natl. Acad. Sci U.S.A. 109, 36993704 (2012)

33. Y. Shao, H. P. Zhao, X. Q. Feng, H. Gao, J. Mech. Phys. Solids 60, 14001419 (2012)

34. F. Barthelat, H. Tang. P. Zavattieri, C. Li, H. Espinosa, J. Mech. Phys. Solids 55, 306337 (2007).

35. R. Z. Wang, Z. Suo, A. G. Evans, N. Yao, L. A. Aksay, J. Mater. Res. 16, 24852493 (2001)

36. R. Rabiei, S. Bekah, F. Barthelat, Acta Biomater. 6, 40814089 (2010).

37. P. Fratzl, O. Kolednik, F. D. Fischer, M. N. Dean, Chem. Soc. Rev. 45, 252267 (2016)

38. S. Deville, Adv. Eng. Mater. 10, 155169 (2008)

\section{ACKNOWLEDGMENTS}

The authors thank Y. Tian, L. Chen, and Y. Guan for computed tomography imaging, and $\mathrm{L}$. Wang for sample preparation. The authors also thank $\mathrm{Y}$. Ni and Z. Song for discussion about mechanical models. This work was supported by the National Natural Science Foundation of China (grant 21431006), the Foundation for Innovative Research Groups of the National Natural Science Foundation of China (grant 21521001), the National Basic Research Program of China (grants 2014CB931800 and 2013CB931800), the Users with Excellence and Scientific Research Grant of Hefei Science Center of CAS (2015HSC UE007 and 2015SRG HSC038), and Key Research Program of Frontier Sciences, CAS (Grant OYZDJ SSW SLH036). Data are available in the supplementary materials. 